\title{
Theoretical and Experimental Investigations of Wave-Induced Vertical Mixing
}

\author{
Wojciech Sulisz and Maciej Paprota \\ Institute of Hydroengineering, Polish Academy of Sciences, Kościerska 7, 80953 Gdańsk, Poland \\ Correspondence should be addressed to Wojciech Sulisz; sulisz@ibwpan.gda.pl
}

Received 12 March 2015; Revised 23 June 2015; Accepted 24 June 2015

Academic Editor: Junwu Wang

Copyright ( 2015 W. Sulisz and M. Paprota. This is an open access article distributed under the Creative Commons Attribution License, which permits unrestricted use, distribution, and reproduction in any medium, provided the original work is properly cited.

\begin{abstract}
Theoretical investigations supported by a series of original laboratory experiments are conducted to study a wave-induced vertical mixing process. The derived semi-analytical solution is very efficient and is applied to predict the effects of water waves on the temperature changes and the evolution of temperature profiles. The results indicate that waves increase a mixing process. The rate of change of the temperature is higher when waves contributed to mixing process and this process increases with increasing the wavelength to water depth ratio. The analysis indicates that for typical ocean waves the contribution of water waves to mixing may be several orders of magnitude higher than a corresponding contribution arising from the classical diffusion process. This implies a need to conduct more theoretical studies and experimental investigation on the effect of water waves on mixing processes. A series of original laboratory experiments were conducted in the insulated wave flume to verify the derived model. The comparisons show a reasonable agreement between predicted and measured temperature profiles. A reasonable agreement between theoretical results and experimental data is observed for the whole considered range of initial temperature distributions. The comparisons indicate that the model is applicable even to cases when gradients in temperature distributions over water depth are fairly high.
\end{abstract}

\section{Introduction}

A wave-induced mixing is an important process from a practical point of view and is a challenging problem for theoretical investigations. The understanding of wave-induced mixing is one of the main tasks of physical oceanographers and climatologists and is of fundamental importance for the modeling and an accurate prediction of ocean transport processes and climate changes.

Water waves are the result of a dynamic process at the intersection of the atmosphere and ocean and constitute one of the largest sources of energy in the ocean [1-3]. Terawatts of energy of water waves imply that waves have a significant effect on ocean processes. In recent decades the influence of waves on transport processes has become even more significant due to increasing number of severe storms occurring mainly in the Northeast Pacific and the North Atlantic [4-6] as a result of climate change $[7,8]$. Theoretical investigations and field observations have shown that waves affect the exchanges of momentum, heat, and masses in the upper oceans and that wave-induced processes lead to vertical mixing in the ocean [9]. The mixing has been attributed to wave breaking process [10-12]. It has been shown that wave energy dissipated through wave breaking enhances turbulence intensity near the sea surface and mixing $[10,13-15]$.

Although it is recognized that waves affect a vertical mixing, the complexity of wave-induced processes in the upper ocean causes that the mixing is attributed to wave breaking phenomena. However, the strong turbulence induced by wave breaking is mainly confined within the near-surface zone with the depth scale of wave heights $[11,16,17]$ and it is hard to justify why this small layer has such a big effect on the upper oceans. It is expected that the whole process is significantly affected by nonbreaking waves which can transport turbulence and increase mixing. Numerical experiments conducted by implementing parameterized wave-induced 
mixing in ocean circulation models and climate models indicate a significant effect of mixing on ocean circulation and climate predictions and a necessity to study mixing induced by nonbraking waves [18-21]. The role of nonbreaking waves in vertical mixing needs further investigations and fundamental research.

Laboratory experiments have been shown to be an efficient method in the investigations of hydrodynamic and thermodynamic problems. Wave-induced mixing processes can be studied in laboratories by conducting experiments in a wave flume. This type of approach has been shown to be indispensable in the studies of many wave-induced processes [22]. The repeatable experiments in the well-controlled environment of a wave flume enable us to perform an accurate investigation that is essential in the analysis of the physics of wave-induced mixing phenomenon. Moreover, a laboratory investigation provides useful data for the analysis of the correlations between spectral and statistical characteristics of wave regimes and wave-induced mixing process. Finally, laboratory experiments enable us to avoid some side effects and separate the mixing originated from breaking and nonbreaking process, which is of fundamental importance for the understanding of mixing processes and an accurate calibration and verification of numerical models.

In this work theoretical studies and laboratory experiments are conducted to investigate a wave-induced mixing process. First, theoretical investigations are conducted to provide insight into a potential origin of a wave-induced mixing. Then, a series of laboratory experiments are conducted to investigate wave-induced vertical mixing. Experimental data are analyzed with the emphasis on factors and wave parameters affecting mixing process. Finally, the theoretical results are compared with experimental data and conclusions are specified.

\section{Theory}

2.1. Particle Kinematics. We consider the propagation of nonlinear water waves in a periodic uniform wave train and their potential effect on wave-induced mixing. A right-hand Cartesian coordinate system is selected such that the $x y$ plane is horizontal and coincides with the undisturbed free surface and $z$ points vertically upwards (Figure 1 ).

The following are assumed:

(i) The fluid is inviscid and incompressible.

(ii) The fluid motion is irrotational.

(iii) The sea bottom is impervious.

According to the assumptions the velocity vector, $\mathbf{V}(x, z, t)$, has a potential $\Phi(x, z, t)$, such that $\mathbf{V}=\nabla \Phi$. The fluid motion is governed by the Laplace equation

$$
\nabla^{2} \Phi=0
$$

and the Bernoulli equation

$$
\Phi_{t}+\frac{1}{\rho} P+g z+\frac{1}{2}|\nabla \Phi|^{2}=0,
$$

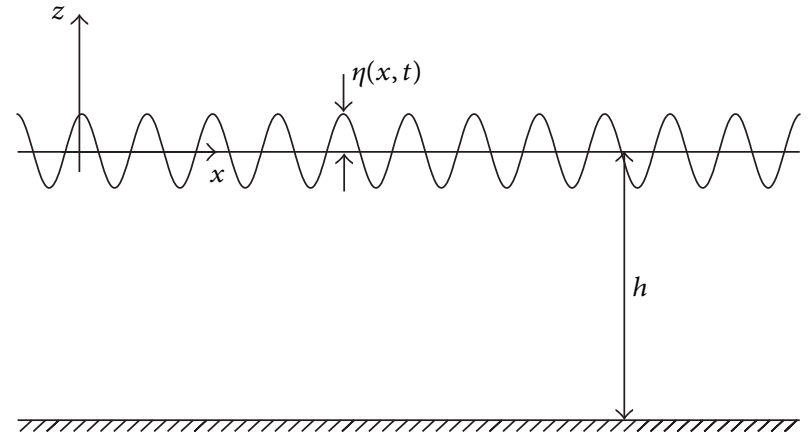

FIGURE 1: Definitions sketch and coordinate systems.

where $\rho$ is the fluid density, $P$ is the pressure, and $g$ is the acceleration due to gravity.

The velocity potential, $\Phi(x, z, t)$, satisfies the Laplace equation

$$
\nabla^{2} \Phi=0
$$

At the free surface the velocity potential, $\Phi(x, z, t)$, has to satisfy the kinematic boundary condition

$$
\eta_{t}+\Phi_{x} \eta_{x}-\Phi_{z}=0, \quad z=\eta(x, t)
$$

and the dynamic boundary condition

$$
\Phi_{t}+g \eta+\frac{1}{2}|\nabla \Phi|^{2}=0, \quad z=\eta(x, t)
$$

At the wavemaker the velocity potential, $\Phi(x, z, t)$, has to satisfy the kinematic boundary condition

$$
\chi_{t}+\Phi_{z} \chi_{z}-\Phi_{x}=0, \quad x=\chi(z, t)
$$

At the sea bottom the following boundary condition must be satisfied:

$$
\Phi_{z}=0, \quad z=-h
$$

Moreover, the velocity potential must satisfy boundary conditions at infinity and initial conditions [23].

A solution of the boundary-value problem, (2a), (2b), $(2 \mathrm{c}),(2 \mathrm{~d})$, and $(2 \mathrm{e})$, is not a trivial task. It is difficult to find the velocity potential which satisfies the free-surface boundary conditions, because the boundary conditions contain nonlinear terms. Additional difficulties arise from a need to apply the boundary conditions on the free surface which is 
unknown and is a part of a final solution [23]. In order to achieve a solution, the kinematic free-surface boundary condition and the dynamic free-surface boundary condition are often expanded in a Taylor series about a mean position:

$$
\begin{aligned}
\sum_{n=0}^{\infty} \frac{\eta^{n}}{n !} \frac{\partial^{n}}{\partial z^{n}}\left(\eta_{t}+\Phi_{x} \eta_{x}-\Phi_{z}\right) & =0, \quad z=0, \\
\sum_{n=0}^{\infty} \frac{\eta^{n}}{n !} \frac{\partial^{n}}{\partial z^{n}}\left(\Phi_{t}+g \eta+\frac{1}{2}|\nabla \Phi|^{2}\right) & =0, \quad z=0, \\
\sum_{n=0}^{\infty} \frac{\chi^{n}}{n !} \frac{\partial^{n}}{\partial x^{n}}\left(\chi_{t}+\Phi_{z} \chi_{z}-\Phi_{x}\right) & =0, \quad x=0,
\end{aligned}
$$

which usually helps to obtain a solution $[24,25]$.

By expanding the kinematic free-surface boundary condition and the dynamic free-surface boundary condition in a Taylor series and collecting terms up to third order in wave amplitude, one obtains the following boundary-value problem:

$$
\begin{aligned}
& \nabla^{2} \Phi=0 \\
& \frac{\partial \eta}{\partial t}+\frac{\partial \Phi}{\partial x} \frac{\partial \eta}{\partial x}-\frac{\partial \Phi}{\partial z}+\eta \frac{\partial^{2} \Phi}{\partial x \partial z} \frac{\partial \eta}{\partial x}-\eta \frac{\partial^{2} \Phi}{\partial z^{2}} \\
& \quad-\frac{1}{2} \eta^{2} \frac{\partial^{3} \Phi}{\partial z^{3}}=0, \quad z=0 \\
& \frac{\partial \Phi}{\partial t}+g \eta+\frac{1}{2}\left(\left(\frac{\partial \Phi}{\partial x}\right)^{2}+\left(\frac{\partial \Phi}{\partial z}\right)^{2}\right)+\eta \frac{\partial^{2} \Phi}{\partial z \partial t} \\
& +\eta\left(\frac{\partial \Phi}{\partial x} \frac{\partial^{2} \Phi}{\partial x \partial z}+\frac{\partial \Phi}{\partial z} \frac{\partial^{2} \Phi}{\partial z^{2}}\right)+\frac{1}{2} \eta^{2} \frac{\partial^{3} \Phi}{\partial z^{2} \partial t}=0 \\
& \frac{\partial \chi}{\partial t}+\frac{\partial \Phi}{\partial z} \frac{\partial \chi}{\partial z}-\frac{\partial \Phi}{\partial x}+\chi \frac{\partial^{2} \Phi}{\partial z \partial x} \frac{\partial \chi}{\partial z}-\chi \frac{\partial^{2} \Phi}{\partial x^{2}} \\
& +\frac{1}{2} \chi \frac{\partial^{3} \Phi}{\partial z \partial x^{2}} \frac{\partial \chi}{\partial z}-\frac{1}{2} \chi^{2} \frac{\partial^{3} \Phi}{\partial x^{3}}=0, \quad x=0, \\
& =0, \quad z=-h .
\end{aligned}
$$

Moreover, the velocity potential must satisfy boundary conditions at infinity and initial conditions [23].

For weakly nonlinear waves the solutions of the boundary-value problem, (4a), (4b), (4c), (4d), and (4e), can be obtained by applying a perturbation method $[26,27]$. The solution is becoming complicated by increasing the number of terms applied in the perturbation method. The basic solu tion may be written in the following form:

$$
\begin{aligned}
& \Phi(x, z, t) \\
& =\frac{A g}{\omega} \frac{\cosh k(z+h)}{\cosh k h} \sin (k x-\omega t) \\
& \quad+\frac{3}{8} \omega A^{2} \frac{\cosh 2 k(z+h)}{\sinh ^{4} k h} \sin 2(k x-\omega t) \\
& \eta(x, t) \\
& =A \cos (k x-\omega t) \\
& \quad+\frac{1}{4} A^{2} k \frac{\cosh ^{3} h}{\sinh ^{3} k h}(2+\cosh 2 k h) \cos 2(k x-\omega t),
\end{aligned}
$$

provided that

$$
\frac{\omega^{2}}{g}=\tanh k h,
$$

where $A$ is the wave amplitude, $k$ is the wave number, $\omega$ is the frequency, and $h$ is the water depth.

For waves of moderate steepness and steep waves a solution of the boundary-value problem, (4a), (4b), (4c), (4d), and (4e), can be obtained by applying a semi-analytical technique. A solution procedure is complicated because of a need to satisfy the kinematic wavemaker boundary condition. In order to obtain a solution the velocity potential, $\Phi$, is expressed as a linear combination of two velocity potentials $\Phi_{f}$ and $\Phi_{w}$. The velocity potential $\Phi_{f}$ is introduced to satisfy the freesurface boundary conditions and the velocity potential $\Phi_{w}$ is introduced to satisfy the wavemaker kinematic boundary condition. As a consequence the solution may be sought in the following form:

$$
\begin{aligned}
& \Phi_{f}(x, z, t)=\sum_{n=0} A_{n} \frac{\cosh \lambda_{n}(z+h)}{\cosh \lambda_{n} h} \cos \lambda_{n} x \\
& \Phi_{w}(x, z, t) \\
& =B_{0}\left[(x-b)^{2}+(z+h)^{2}\right] \\
& \quad+\sum_{m=1} B_{m} \frac{\cosh \mu_{m}(x-b)}{\cosh \mu_{m} b} \cos \mu_{m}(z+h) \\
& \eta(x, t)=\sum_{n=0} a_{n} \cos \lambda_{n} x,
\end{aligned}
$$

where

$$
\begin{aligned}
& \lambda_{n}=\frac{n \pi}{b}, \\
& \mu_{m}=\frac{m \pi}{h},
\end{aligned}
$$

in which $A_{n}, B_{m}$, and $a_{n}$ are functions of time and $b$ is the length of a computational domain. The solution in the form of the eigenfunction expansions, (6a), (6b), (6c), (6d), and (6e), 
satisfies the Laplace equation and the bottom boundary condition. A time-stepping procedure is applied to satisfy the remaining boundary conditions (4b) and (4c) and to determine the unknown coefficients of the eigenfunction expansions. The time-stepping procedure is based on the AdamsBashforth-Moulton predictor-corrector method [28]. The method enables prediction of the value of a function $f$ from its time derivatives $f^{\prime}$. According to the solution procedure, the Adams-Bashforth predictor

$$
f_{n+1}=f_{n}+\frac{\Delta t}{24}\left[55 f_{n}^{\prime}-59 f_{n-1}^{\prime}+37 f_{n-2}^{\prime}-9 f_{n-3}^{\prime}\right]
$$

is combined with the Adams-Moulton corrector

$$
f_{n+1}=f_{n}+\frac{\Delta t}{24}\left[9 f_{n+1}^{\prime}+19 f_{n}^{\prime}-5 f_{n-1}^{\prime}+f_{n-2}^{\prime}\right]
$$

and with the free-surface boundary conditions (4b) and (4c) to predict the free-surface elevation $\eta$ and the velocity potential $\Phi$ at a new time step.

2.2. Modeling of Mixing. The equation for temperature diffusion can be written in the following form:

$$
\frac{\partial T}{\partial t}-\frac{\partial}{\partial z}\left(\kappa \frac{\partial T}{\partial z}\right)=0
$$

where $T$ denotes the water temperature, $\kappa$ is the vertical diffusion coefficient, $t$ is the time, and $z$ is the vertical coordinate.

A theoretical analysis indicates that the vertical diffusion coefficient may be written in the form of a sum of the molecular and wave-induced diffusivities:

$$
\kappa=\kappa_{m}+\kappa_{V},
$$

where $\kappa_{m}=1.4 \cdot 10^{-7} \mathrm{~m}^{2} / \mathrm{s}$ is the background molecular diffusivity and $\kappa_{V}$ represents the wave-induced diffusivity.

There are several possibilities to assess $\kappa_{V}$. In the present studies the Prandtl mixing length hypothesis is applied to calculate the wave-induced diffusivity. By applying the Prandtl mixing length approach the wave-induced diffusivity may be calculated from

$$
\kappa_{V}=\left\langle l w^{\prime}\right\rangle
$$

where $l$ is the mixing length and $w^{\prime}$ is the increment of the vertical velocity.

Since the increment of the velocity in the vertical direction can be expressed as

$$
w^{\prime}=l \frac{\partial\langle w w\rangle^{0.5}}{\partial z}
$$

the formula for the wave-induced diffusivity may be written in the following form:

$$
\kappa_{V}=\left\langle l^{2}\right\rangle \frac{\partial\langle w w\rangle^{0.5}}{\partial z}
$$

For weakly nonlinear waves it is reasonable to assume that the mixing length $l$ is proportional to the particle displacement in the vertical direction. Since the mixing length $l$ is proportional to the wave particle displacement, the product $\left\langle l^{2}\right\rangle$ may be written as follows:

$$
\left\langle l^{2}\right\rangle \propto A^{2} \frac{\sinh ^{2} k(z+h)}{\sinh ^{2} k h} .
$$

Finally, by substituting (13) into (12) and conducting simple derivations, the formula for the wave-induced diffusivity may be written as

$$
\kappa_{V}=\alpha A^{3} k \omega \frac{\sinh ^{2} k(z+h) \cosh k(z+h)}{\sinh ^{3} k h},
$$

where $\alpha$ is the dimensionless coefficient which should be determined by observations or numerical experiments. In previous studies, due to the lack of information on the value of this coefficient, its value was assumed to be 1 by default. In Figure 2 the wave-induced diffusivity calculated using (14) for different wave cases is presented.

For waves of moderate steepness and steep waves the wave-induced diffusivity was calculated by applying the derived nonlinear wave model and the formula (12). The mean values in (12) of the square of wave particle displacement and vertical derivative of square root of the product of vertical velocity component were calculated by integrating these quantities over a finite number of wave periods of regular waves. In Figure 3 the wave-induced diffusivity calculated for waves of moderate steepness and steep waves for different wave cases is presented.

The plots in Figures 2 and 3 show that for waves of small and moderate steepness nonlinear effects are of secondary importance and a weakly nonlinear wave theory can be applied to determine a wave-induced diffusivity. For steeper waves, nonlinear wave effects and the effects arising from the interaction of wave components are becoming more and more important and a nonlinear approach must be applied to calculate a wave-induced diffusivity.

The closed-form expression derived for the wave-induced diffusivity enables us to incorporate water waves in a mixing process. As a consequence, the temperature in the water column and its evolution must satisfy

$$
\frac{\partial T}{\partial t}-\frac{\partial}{\partial z}\left(\kappa \frac{\partial T}{\partial z}\right)=0,
$$

at the free water surface

$$
\nu T-\frac{\partial T}{\partial z}=0, \quad z=0
$$

and at the bottom

$$
\frac{\partial T}{\partial z}=0, \quad z=-h
$$

provided that

$$
T(z)=T_{0}(z), \quad t=0,
$$

where $T_{0}(z)$ is assumed to be the known temperature distribution in the water column at $t=0$. The condition at $z=0$ 

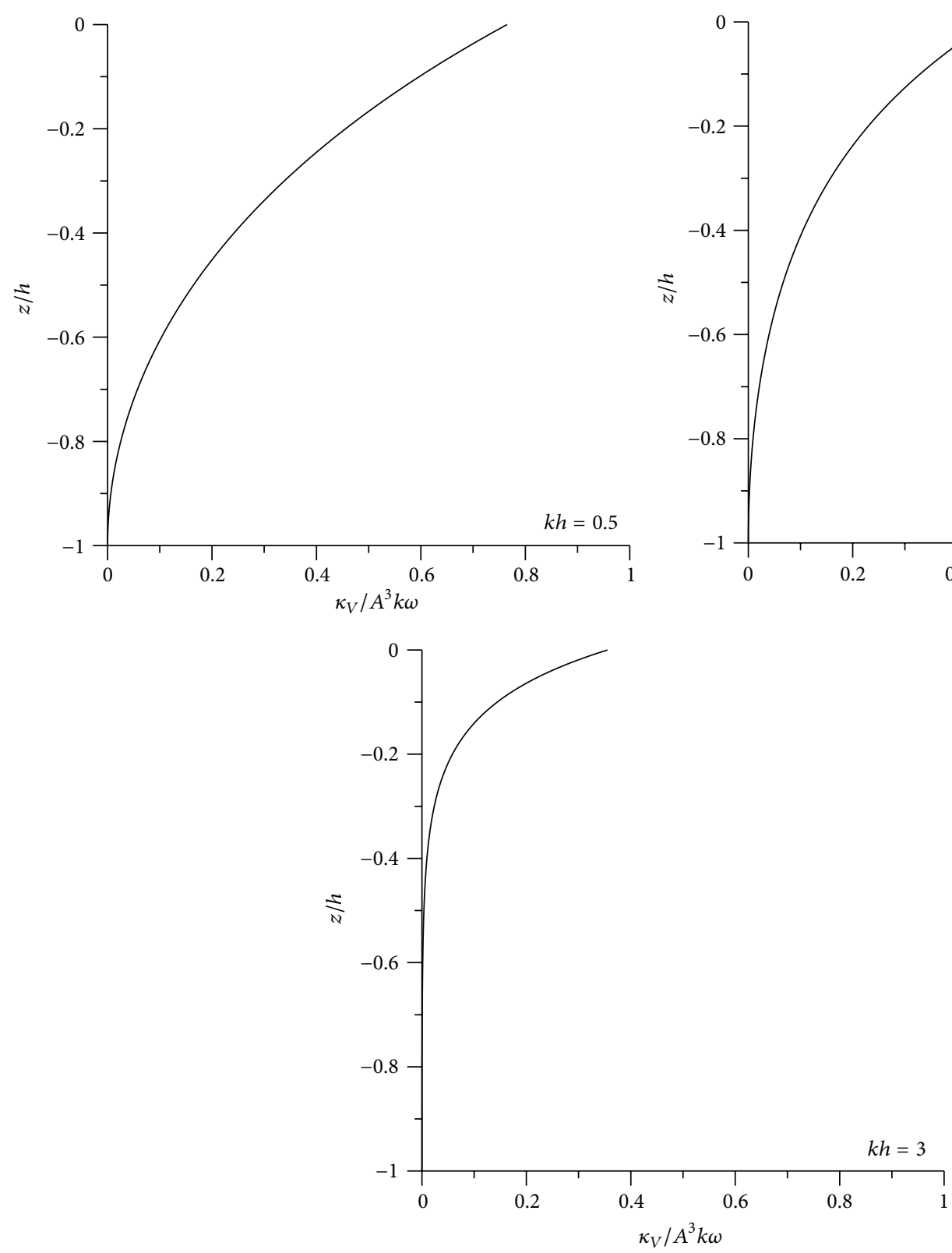

FIGURE 2: Wave-induced diffusivity for weakly nonlinear waves.

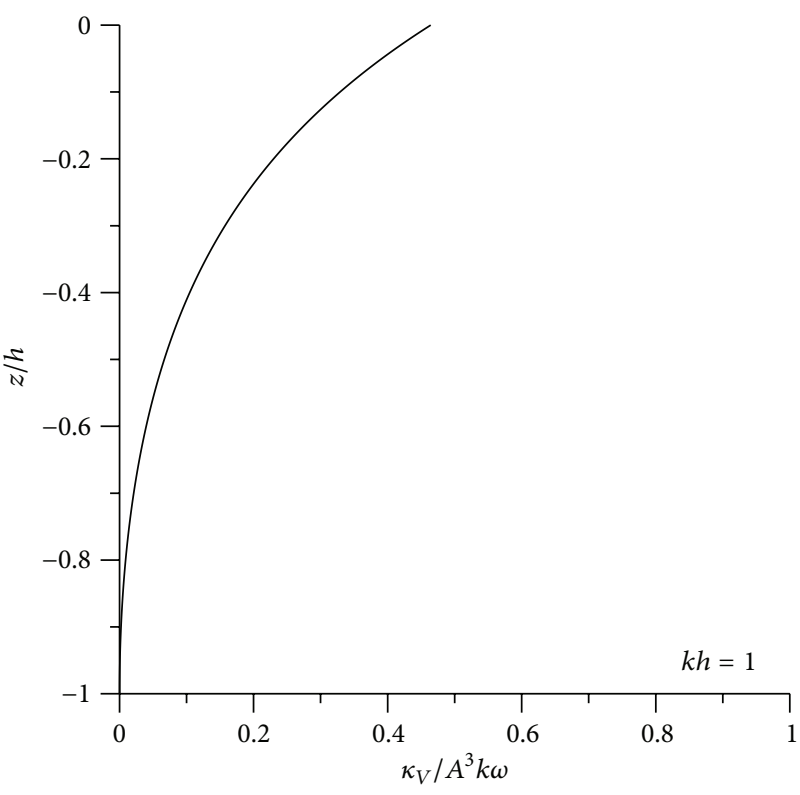

accounts for a partial radiation at the water surface. This type of conditions is widely applied in the modelling of many physical phenomena. In general, $v$ may be a complex-value quantity, which often facilitates modelling process.

The solution of (15a), (15b), (15c), and (15d) is achieved by a time-stepping procedure and applying an eigenfunction expansion method to describe $T$ and the Adams-BashforthMoulton formulas to proceed in time [28]. This method has been shown to be an efficient technique in the modeling of the propagation and transformation of nonlinear water waves $[22,29]$. Accordingly, the solution has the following form:

$$
T=\sum_{n=1}^{N} a_{n}(t) \cos \lambda_{n}(z+h)
$$

provided that the eigenvalues satisfy

$$
v+\lambda_{n} \tan \left(\lambda_{n} h\right)=0
$$

The coefficients of the eigenfunction expansions are determined by applying a Fourier method. The application of the eigenfunction expansions and a Fourier method makes the solution procedure a very efficient technique and enables us to obtain results even for large spatial or time domains.

The wave-induced diffusivity, (14), has been derived for the first time and enables us to eventually evaluate the role of surface waves in mixing process. The derivation is based on the Prandtl mixing length hypothesis. Because of uncertainties related with possibilities to apply the Prandtl mixing 

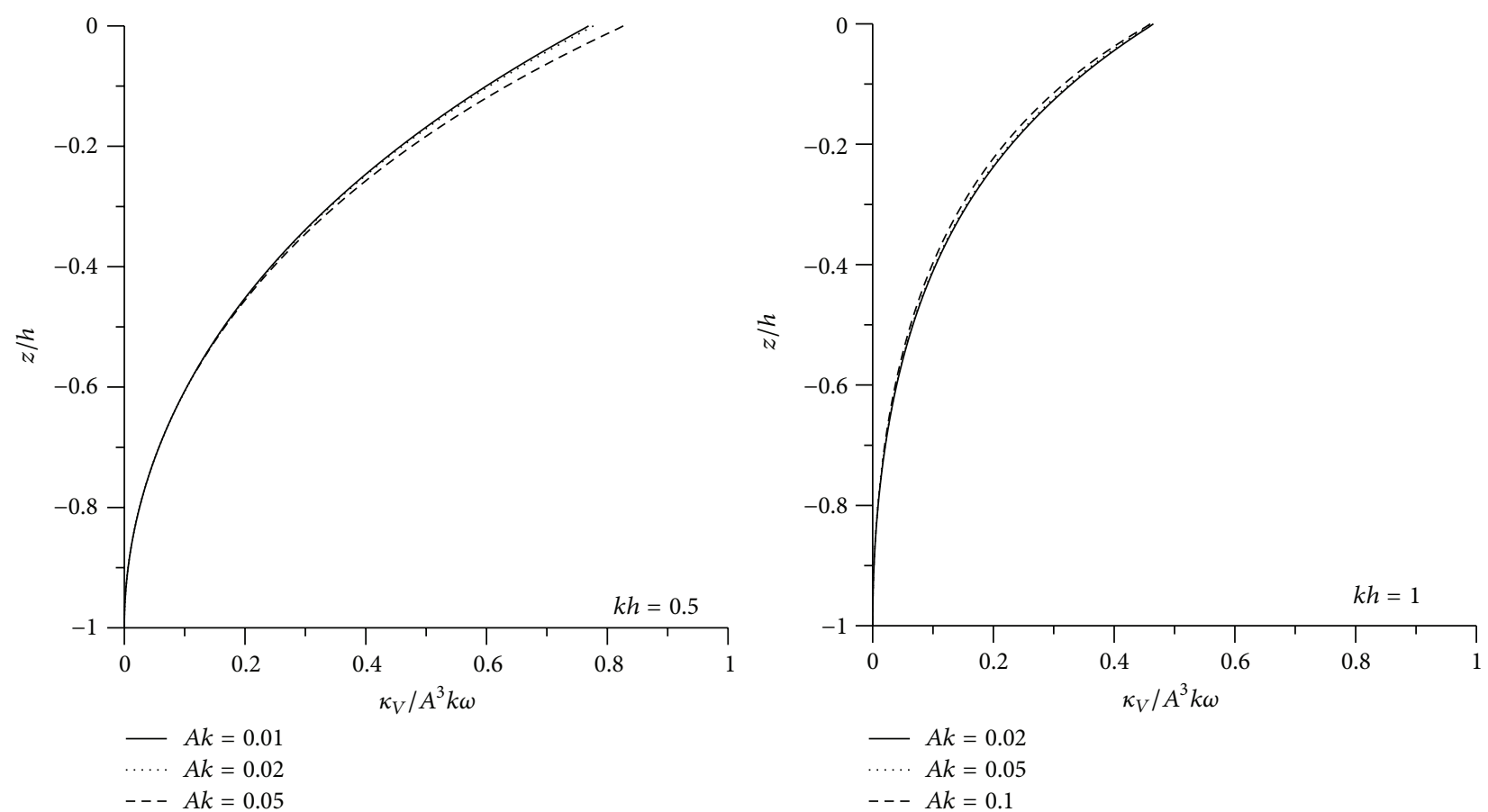

- $A k=0.02$

… $A k=0.05$

$---A k=0.1$

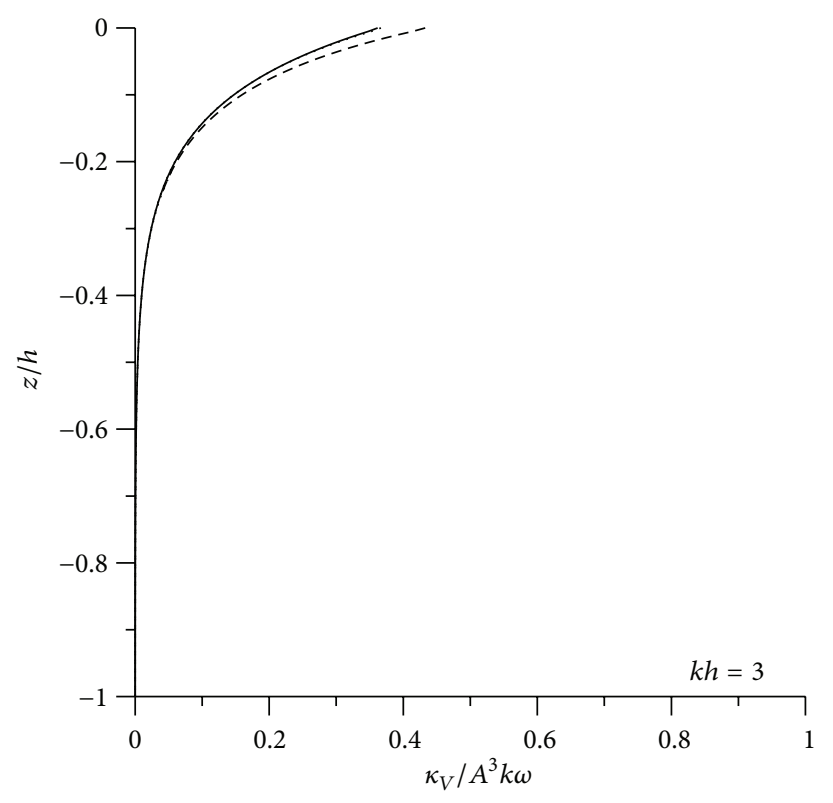

$\begin{array}{rl}\text { —. } & A k=0.02 \\ \ldots \ldots k & A k=0.05 \\ \ldots- & A k=0.1\end{array}$

FIGURE 3: Wave-induced diffusivity for waves of moderate steepness and steep waves at $x / h=6$.

length hypothesis to adequately describe the wave-induced diffusivity and the lack of solid evidence on its applicability range, experimental verification of the proposed model seems to be a must. This is why in this study laboratory experiments are conducted to provide insight into mixing process and eventually supply experimental data to verify the derived model.

\section{Laboratory Experiments}

3.1. Wave Flume. Laboratory experiments were conducted in the wave flume at the Institute of Hydroengineering, Polish Academy of Sciences, Gdańsk. The wave flume at the Institute of Hydroengineering is $64 \mathrm{~m}$ long and $1.4 \mathrm{~m}$ deep. It is equipped with a programmable piston wave generator. 


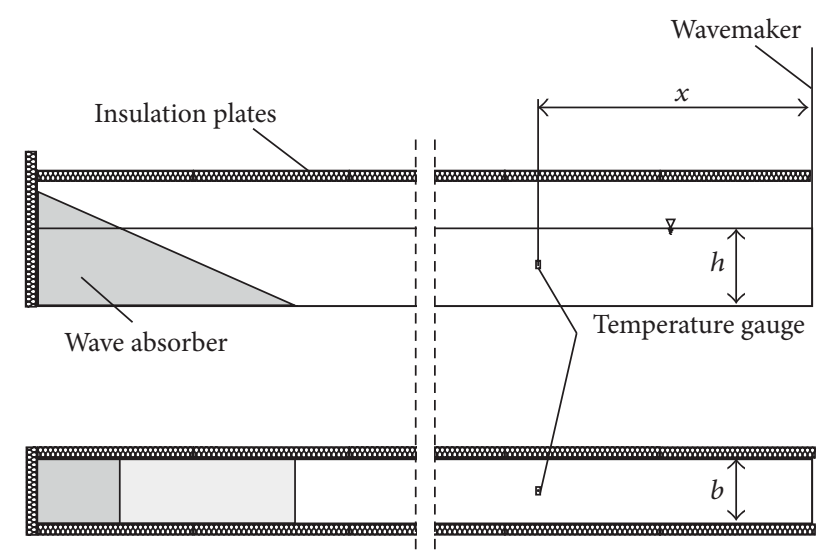

FIGURE 4: Side and top view of the wave flume.

The experiments were performed in a separated section of the wave flume of the width $b=0.3 \mathrm{~m}$ shown schematically in Figure 4 . The water depth in the wave flume was $h=0.4 \mathrm{~m}$. A system of electrical heaters was immersed into the upper water layer and the water was heated up. Then, the system of heaters was lifted and the flume was fully covered by insulation plates. The temperature was measured by a temperature gauge. The temperature sensor was installed on a measuring rod to precisely identify its vertical position. The measurements were performed with the maximum absolute error of $0.1^{\circ} \mathrm{C}$ for temperature registration and $0.0001 \mathrm{~m}$ for location. The distance from the temperature gauge to the wavemaker was $x=1.3 \mathrm{~m}$. Final experiments and temperature records were preceded by a series of control measurements in the horizontal and vertical directions.

In the first series of laboratory experiments investigations focused on temperature diffusion for still water conditions. Laboratory experiments were performed in the insulated wave flume shown schematically in Figure 4 . A system of electrical heaters was immersed $0.12 \mathrm{~m}$ into the upper water layer and the water was heated up. Then, the temperature profile over depth was measured. The measurements were conducted every $0.01 \mathrm{~m}$. After approximately one hour the temperature profile was measured again to record changes of temperature due to diffusion and heat losses. The experiments were repeated for two different initial temperature profiles to provide more data for the verification of the derived model. In the first case the measurements started when the temperature at the free surface reached $33.2^{\circ} \mathrm{C}$ and in the second case, intended to include profiles of lower temperature gradients, the measurements started at $27.8^{\circ} \mathrm{C}$.

In the second series of laboratory experiments the investigations focused on the effect of surface waves on the vertical mixing process. Laboratory experiments were performed in the same insulated section of the wave flume as previous series of experiments. A system of electrical heaters was used to heat up the upper water layer in the wave flume. Then, the temperature profile over depth was measured. The measurements were conducted every $0.01 \mathrm{~m}$. After the measurement of the temperature profile was finished, a train of regular waves was generated. When waves and internal waves decayed, the temperature profile over depth was measured again to provide information on the effect of surface waves on vertical mixing process. The experiments were repeated for two different scenarios to provide more data for the verification of the derived model. In the first case the wavemaker generated deep-water waves for $105 \mathrm{~s}$ with $A_{1} / h=0.065$, $L / h=2$ and in the second case waves were generated for $120 \mathrm{~s}$ with $A_{1} / h=0.07, L / h=4$. Water waves considered in the first case are typical deep-water waves. Waves in the second case, higher and longer, were included in the experimental program intentionally, because these waves were expected to have more pronounced effect on mixing than waves in the first case.

\section{Results}

The results corresponding to the first series of laboratory experiments conducted for still water conditions are presented in Figures 5 and 6 . The plots show experimental data of the initial and final temperature distribution over depth, initial temperature applied in the derived diffusion model, and the comparisons between theoretical results and experimental data for the final temperature distribution.

The results in Figures 5 and 6 show that the temperature in the water column decreases in the upper water layer and increases in its lower part, as expected. The rate of change of the temperature is higher in the upper layer which is related with higher gradients of temperature in this layer. The derived model predicts fairly well the initial and final temperature distribution in the water column. A reasonable agreement between theoretical results and experimental data is observed for both considered cases of initial temperature distributions. The comparisons indicate that the model is applicable even to cases when gradients in temperature distributions over water depth are fairly high.

In the second series of laboratory experiments regular wave trains were generated by the wavemaker to study the effect of surface waves on the vertical mixing process. The results are presented in Figures 7 and 8 . The plots show experimental data of the initial temperature distribution and the final distribution of temperature over depth caused by 


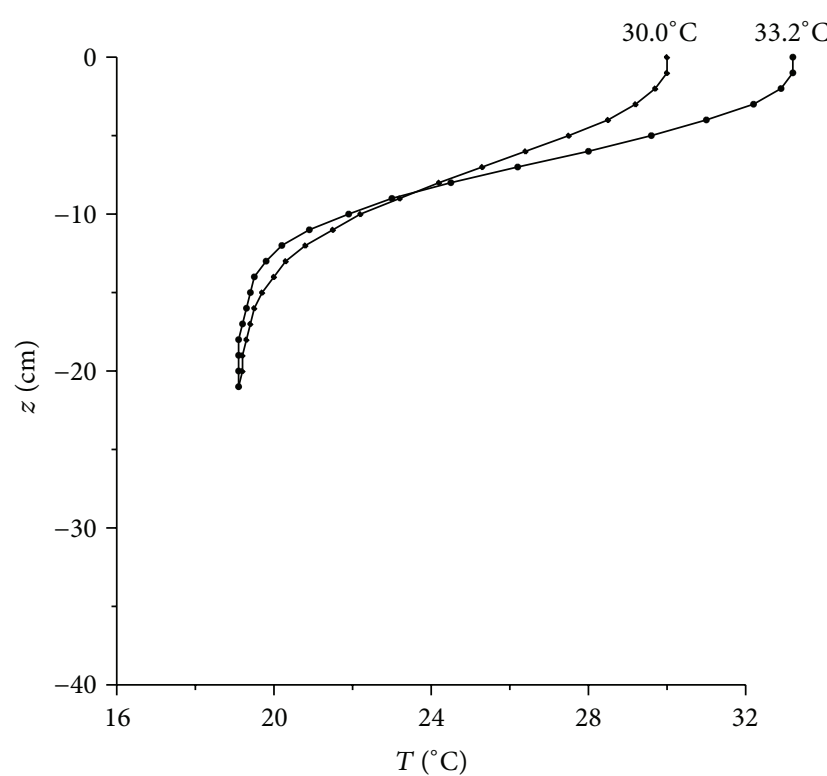

(a)

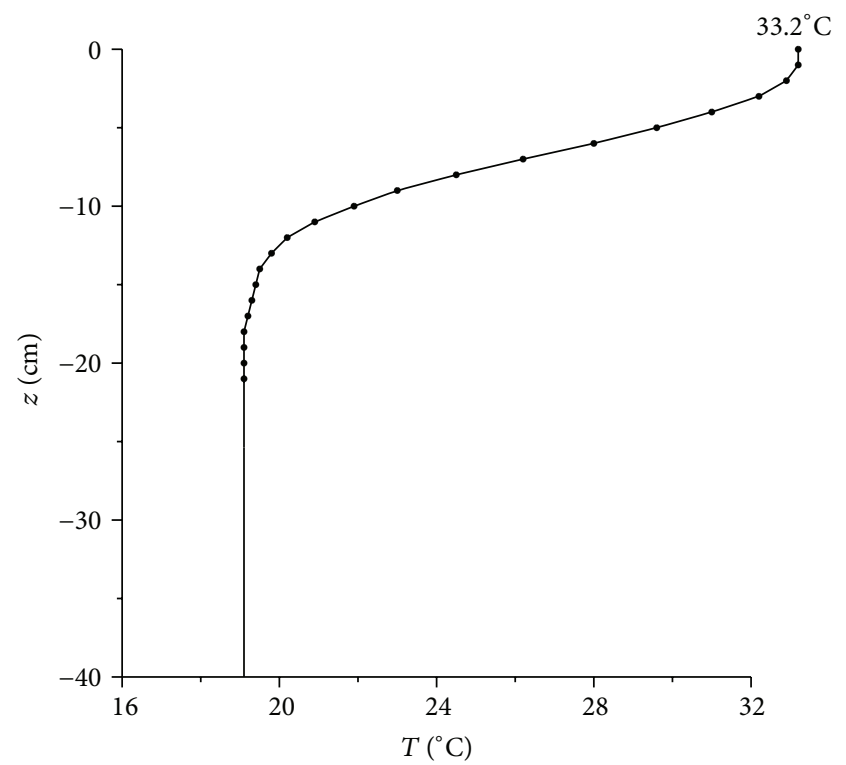

(b)

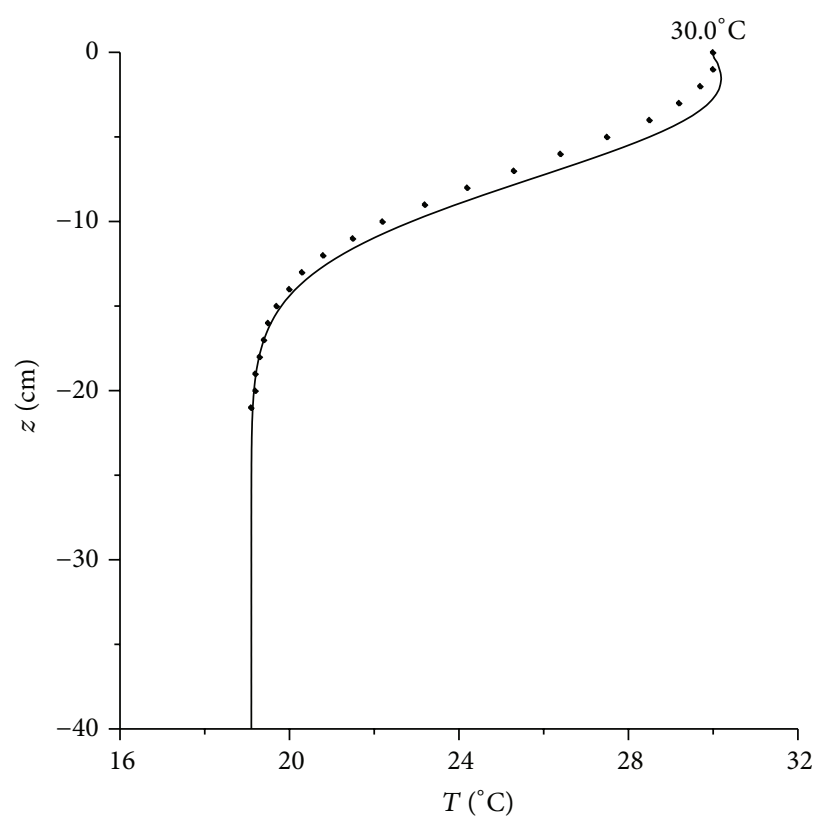

(c)

FIGURE 5: (a) Recorded initial and final temperature distribution for still water conditions, $t=3600 \mathrm{~s}$. (b) Initial temperature distribution for still water conditions, $\bullet$ - experimental data, - model. (c) Final temperature distribution for still water conditions, $\bullet$ ・ experimental data, - model.

diffusion process and wave-induced mixing, initial temperature distribution applied in the derived model, and the comparisons between theoretical results and experimental data for the final temperature distribution. The comparisons were conducted for $\alpha=0.002$ that was assessed based on results corresponding to waves generated in the first case.

The results in Figures 7 and 8 show that the temperature in the water column decreases in the upper water layer and increases in its lower part. The plots show that the rate of change of the temperature is higher in the upper layer than at the lower water levels. The results indicate that waves increase mixing process. The rate of change of the temperature is higher when waves contributed to mixing process and this process increases with increasing the wavelength to water depth ratio. The derived model predicts fairly well the initial and final temperature distribution in the water column. A reasonable agreement between theoretical results and experimental data is observed for both considered cases of initial 


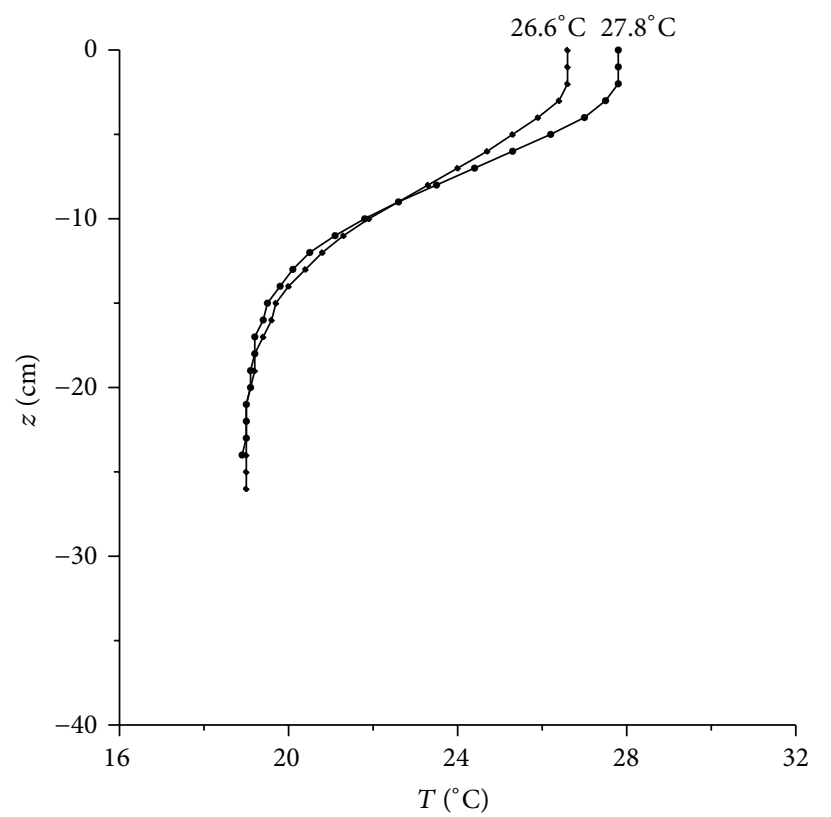

(a)

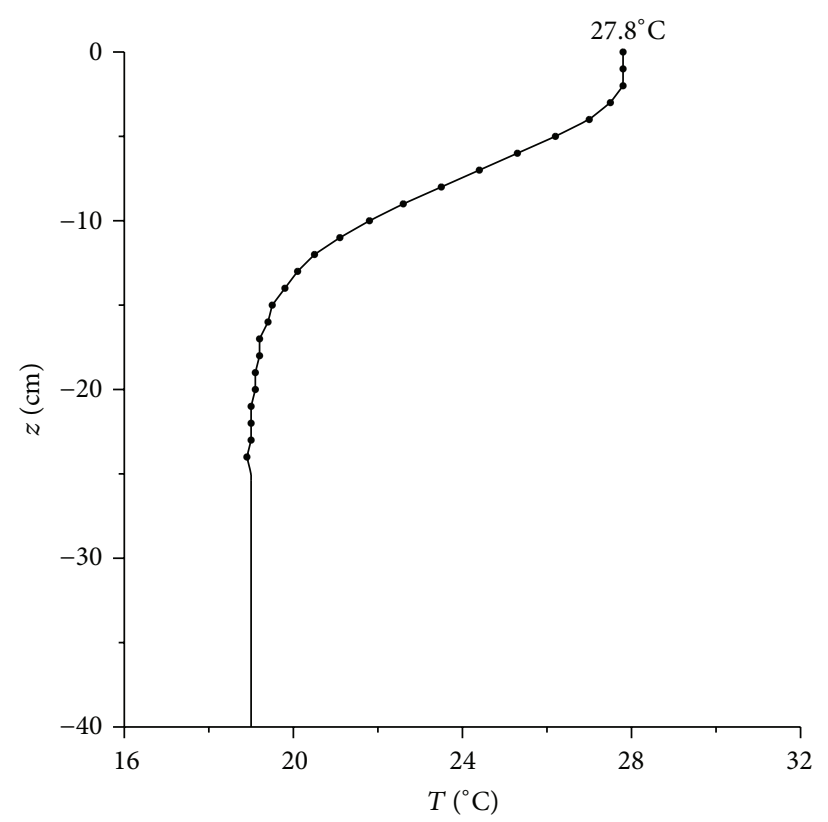

(b)

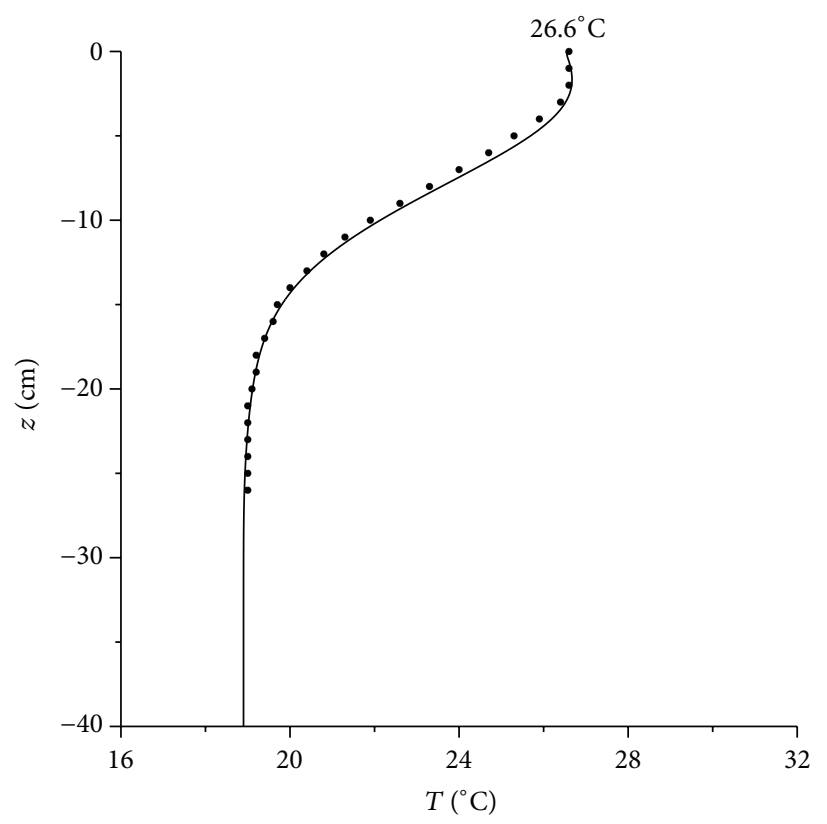

(c)

FiguRE 6: (a) Recorded initial and final temperature distribution for still water conditions, $t=2700 \mathrm{~s}$. (b) Initial temperature distribution for still water conditions, $\cdots$ - experimental data, — model. (c) Final temperature distribution for still water conditions, $\cdots$ - experimental data, - model.

temperature distributions. The comparisons indicate that the model is applicable even to cases when gradients in temperature distributions over water depth are fairly high.

A fairly good agreement obtained between theoretical results and experimental data and the analysis of (14) indicate that for typical ocean waves, which are about two orders of magnitude higher than waves considered in laboratory experiments, the contribution of water waves to mixing may be several orders of magnitude higher than a corresponding contribution arising from a diffusion process. This implies a need to conduct more theoretical studies and experimental investigation on the effect of water waves on mixing processes. 


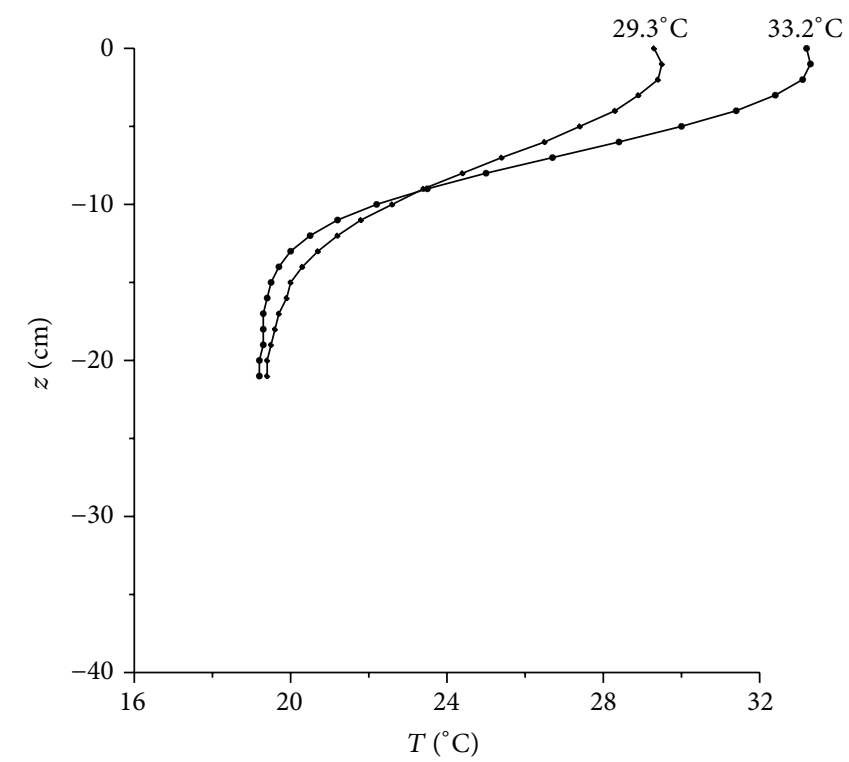

(a)

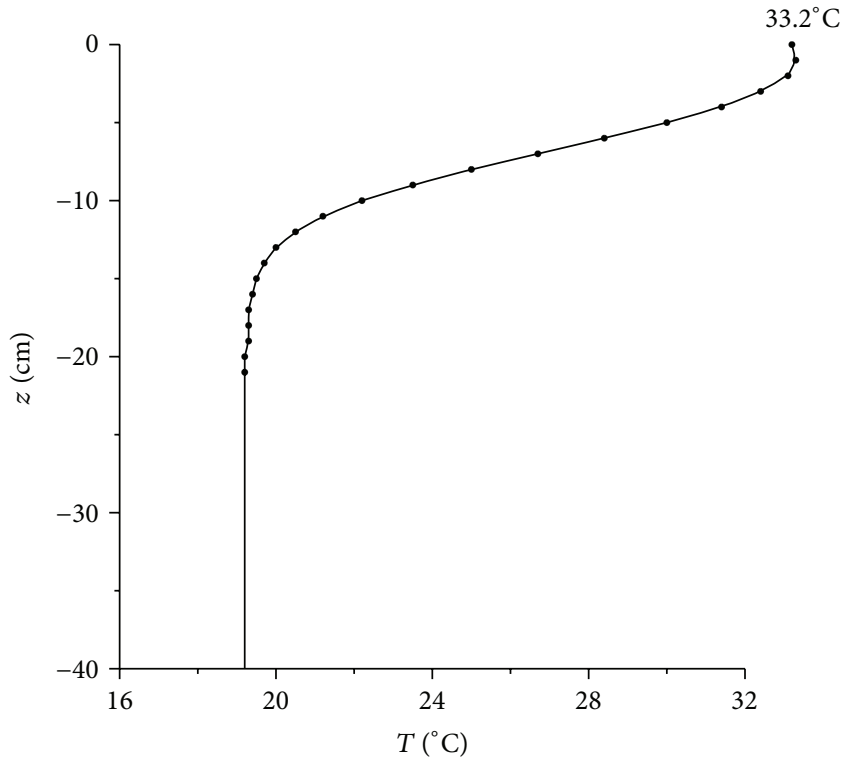

(b)

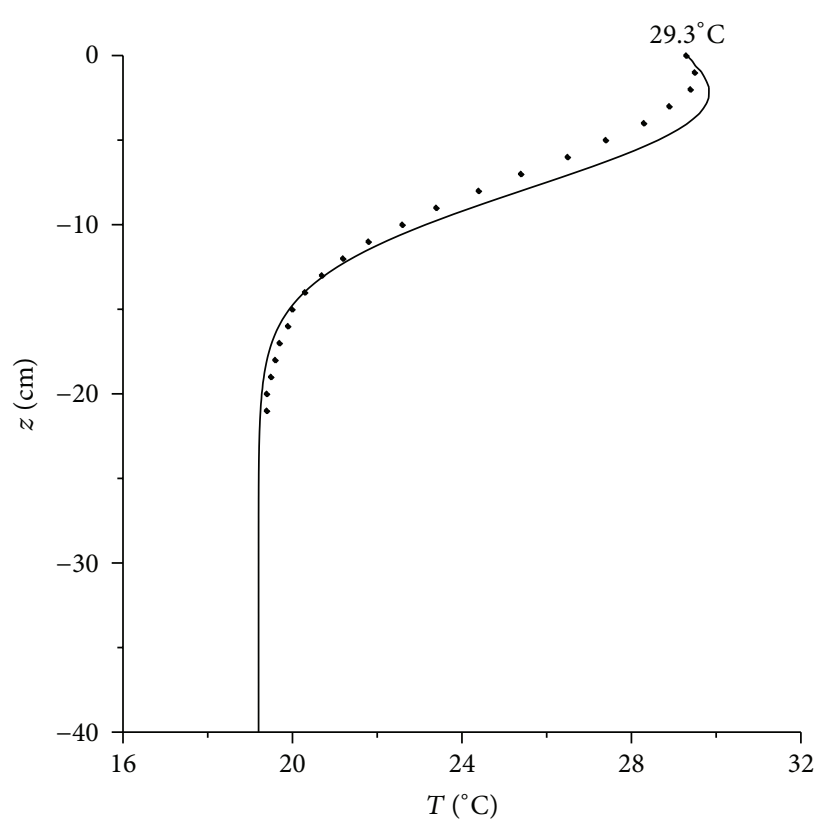

(c)

Figure 7: (a) Recorded initial and final temperature distribution for mixing affected by waves of $L / h=2, t=3600 \mathrm{~s}$. (b) Initial temperature distribution for mixing affected by waves of $L / h=2, \cdots \bullet$ experimental data, - model. (c) Final temperature distribution for mixing affected by waves of $L / h=2, \cdots \bullet$ experimental data, - model.

\section{Summary}

Theoretical investigations supported by a series of original laboratory experiments are conducted to study a waveinduced vertical mixing process. An initial boundary-value problem is formulated to describe the evolution of mixing and is solved by applying eigenfunction expansions and a time-stepping procedure. The derived semi-analytical solution is very efficient and is applied to predict the effects of water waves on the temperature changes and the evolution of temperature profiles.

The results show that the temperature in the water column decreases in the upper water layer and increases in its lower part, as expected. The rate of change of the temperature is higher in the upper layer, which is related with higher gradients of temperature in this layer. The results indicate that waves increase mixing process. The rate of change of the 


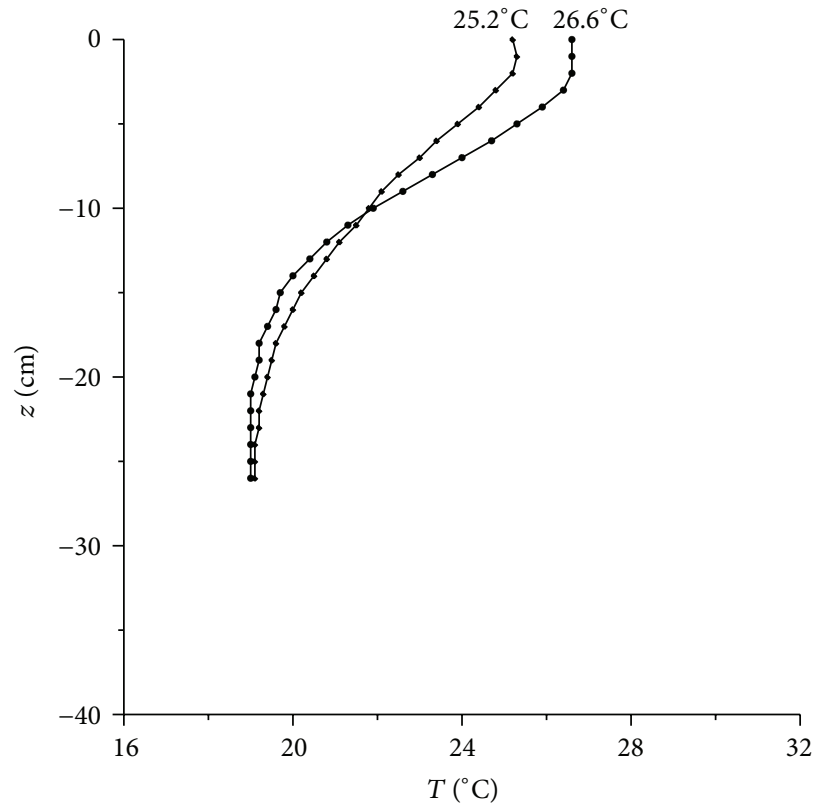

(a)

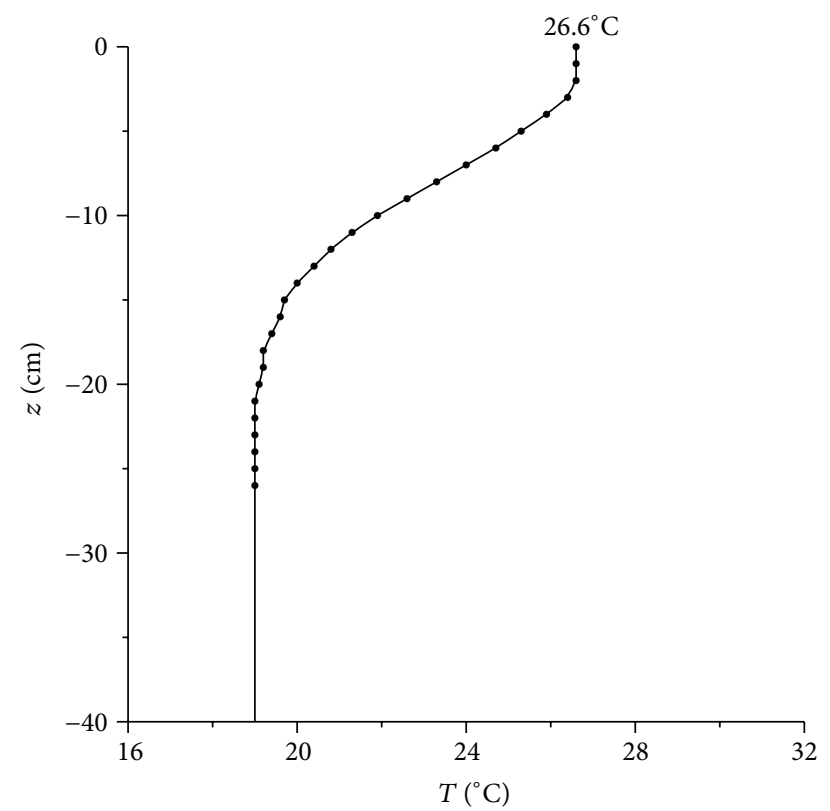

(b)

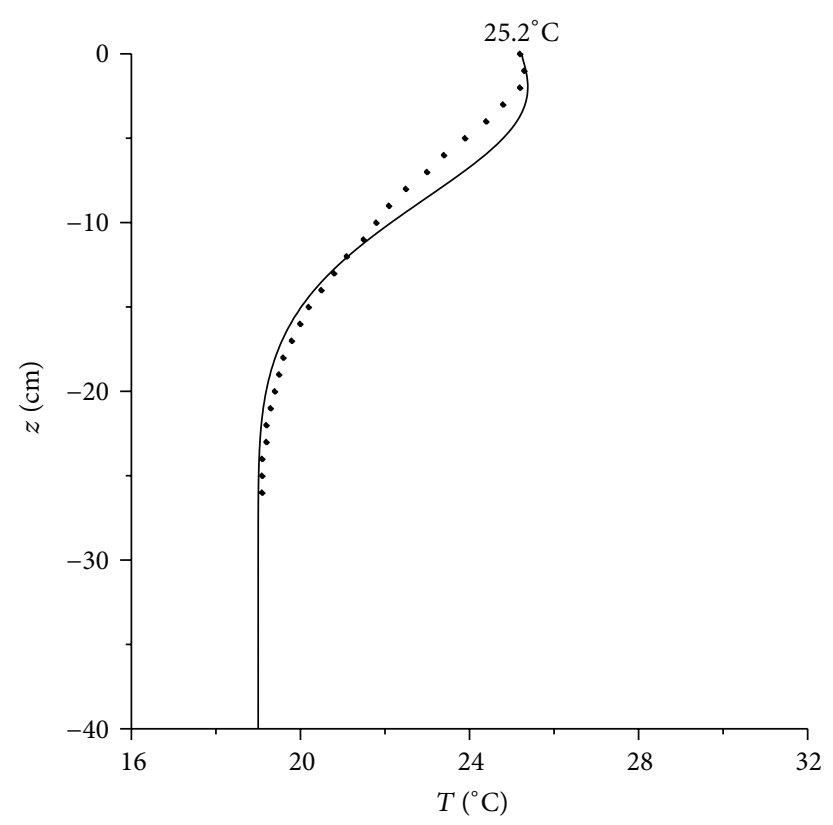

(c)

FIGURE 8: (a) Recorded initial and final temperature distribution for mixing affected by waves of $L / h=4, t=3000 \mathrm{~s}$. (b) Initial temperature distribution for mixing affected by waves of $L / h=4, \cdots \bullet$ experimental data, - model. (c) Final temperature distribution for mixing affected by waves of $L / h=4, \cdots$ experimental data, - model.

temperature is higher when waves contributed to mixing process and this process increases with increasing the wavelength to water depth ratio.

The analysis indicates that for typical ocean waves the contribution of water waves to mixing may be several orders of magnitude higher than a corresponding contribution arising from a diffusion process. This implies a need to conduct more theoretical studies and experimental investigation on the effect of water waves on mixing processes.

A series of original laboratory experiments were conducted in the insulated wave flume to verify the derived model. The comparisons show a reasonable agreement between predicted and measured temperature profiles. A reasonable agreement between theoretical results and experimental data 
is observed for the whole considered range of initial temperature distributions. The comparisons indicate that the model is applicable even to cases when gradients in temperature distributions over water depth are fairly high.

\section{Conflict of Interests}

The authors declare that there is no conflict of interests regarding the publication of this paper.

\section{Acknowledgments}

The assistance of Professor W. Chybicki and Dr. A. Reda from the Institute of Hydroengineering, PAS, Gdańsk, in conducting laboratory experiments is appreciated. The first author is indebted to Professor F. Qiao and Professor D. Dai from the First Institute of Oceanography, SOA, Qingdao, for their enthusiastic support to the proposal of verifying waveinduced mixing hypotheses by conducting laboratory experiments.

\section{References}

[1] R. Ferrari and C. Wunsch, "Ocean circulation kinetic energy: reservoirs, sources, and sinks," Annual Review of Fluid Mechanics, vol. 41, pp. 253-282, 2009.

[2] W. Wang and R. X. Huang, "Wind energy input to the surface waves," Journal of Physical Oceanography, vol. 34, no. 5, pp. 1276-1280, 2004

[3] N. Rascle, F. Ardhuin, P. Queffeulou, and D. Croizé-Fillon, "A global wave parameter database for geophysical applications. Part 1: wave-current-turbulence interaction parameters for the open ocean based on traditional parameterizations," Ocean Modelling, vol. 25, no. 3-4, pp. 154-171, 2008.

[4] X. L. Wang and V. R. Swail, "Changes of extreme wave heights in Northern Hemisphere oceans and related atmospheric circulation regimes," Journal of Climate, vol. 14, no. 10, pp. 2204-2221, 2001.

[5] P. Ruggiero, P. D. Komar, and J. C. Allan, "Increasing wave heights and extreme value projections: the wave climate of the U.S. Pacific Northwest," Coastal Engineering, vol. 57, no. 5, pp. 539-552, 2010.

[6] R. J. Seymour, "Evidence for changes to the northeast Pacific wave climate," Journal of Coastal Research, vol. 27, no. 1, pp. 194201, 2011.

[7] H. Gunther, W. Rosenthal, M. Stawarz et al., "The wave climate of the Northeast Atlantic over the period 1955-94: the WASA wave hindcast," Global Atmosphere and Ocean System, vol. 6, pp. 121-163, 1998

[8] P. D. Komar, J. C. Allan, and P. Ruggiero, "Ocean wave climates: trends and variations due to Earth's changing climate," in Handbook of Coastal and Ocean Engineering, Y. C. Kim, Ed., pp. 971975, World Scientific Publishing, 2009.

[9] T. Matsuno, J.-S. Lee, M. Shimizu, S.-H. Kim, and I.-C. Pang, "Measurements of the turbulent energy dissipation rate and an evaluation of the dispersion process of the Changjiang Diluted Water in the East China Sea," Journal of Geophysical Research, vol. 111, Article ID C11S09, 2006.

[10] Y. C. Agrawal, E. A. Terray, M. A. Donelan et al., "Enhanced dissipation of kinetic energy beneath surface waves," Nature, vol. 359, no. 6392, pp. 219-220, 1992.
[11] P. D. Craig and M. L. Banner, "Modeling wave-enhanced turbulence in the ocean surface layer," Journal of Physical Oceanography, vol. 24, no. 12, pp. 2546-2559, 1994.

[12] E. A. Terray, M. A. Donelan, Y. C. Agrawal et al., "Estimates of kinetic energy dissipation under breaking waves," Journal of Physical Oceanography, vol. 26, no. 5, pp. 792-807, 1996.

[13] M. A. Donelan, "Air-water exchange processes," in Physical Processes in Lakes and Oceans, J. Imberger, Ed., vol. 54 of Coastal and Estuarine Studies, pp. 19-36, American Geophysical Union, Washington, DC, USA, 1998.

[14] W. M. Drennan, M. A. Donelan, E. A. Terray, and K. B. Katsaros, "Oceanic turbulence dissipation measurements in SWADE," Journal of Physical Oceanography, vol. 26, no. 5, pp. 808-815, 1996.

[15] H. Burchard, "Simulating the wave-enhanced layer under breaking surface waves with two-equation turbulence models," Journal of Physical Oceanography, vol. 31, no. 11, pp. 3133-3145, 2001.

[16] R. J. Rapp and W. K. Melville, "Laboratory measurements of deep-water breaking waves," Philosophical Transactions of the Royal Society A: Mathematical, Physical and Engineering Sciences, vol. 331, no. 1622, pp. 735-800, 1990.

[17] A. Soloview and R. Lukas, "Observation of wave-enhanced turbulence in the near-surface layer of the ocean during TOGA COARE," Deep-Sea Research I, vol. 50, pp. 371-395, 2003.

[18] F. Qiao, Y. Yuan, Y. Yang, Q. Zheng, C. Xia, and J. Ma, "Waveinduced mixing in the upper ocean: distribution and application to a global ocean circulation model," Geophysical Research Letters, vol. 31, no. 11, Article ID L11303, 2004.

[19] C. Xia, F. Qiao, Y. Yang, J. Ma, and Y. Yuan, "Three-dimensional structure of the summertime circulation in the Yellow Sea from a wave-tide-circulation coupled model," Journal of Geophysical Research, vol. 111, no. 11, 2006.

[20] Z. Song, F. Qiao, Y. Yang, and Y. Yuan, "An improvement of the too cold tongue in the tropical Pacific with the development of an ocean-wave-atmosphere coupled numerical model," Progress in Natural Science, vol. 17, no. 5, pp. 576-583, 2007.

[21] C. Huang, F. Qiao, and Z. Song, "The effect of the wave-induced mixing on the upper ocean temperature in a climate model," Acta Oceanologica Sinica, vol. 27, no. 3, pp. 104-111, 2008.

[22] W. Sulisz and M. Paprota, "Modeling of the propagation of transient waves of moderate steepness," Applied Ocean Research, vol. 26, no. 3-4, pp. 137-146, 2004.

[23] J. V. Wehausen, "Surface waves," in Handbuch der Physik, vol. 9, pp. 446-778, Springer, Berlin, Germany, 1960.

[24] R. G. Dean and R. T. Dalrymple, Water Wave Mechanics for Engineers and Scientists, Prentice-Hall, 1984.

[25] R. T. Hudspeth and W. Sulisz, "Stokes drift in two-dimensional wave flumes," Journal of Fluid Mechanics, vol. 230, pp. 209-229, 1991.

[26] B. Kinsman, Wind Waves, Prentice-Hall, Englewood Cliffs, NJ, USA, 1965.

[27] W. Sulisz and R. T. Hudspeth, "Complete second-order solution for water waves generated in wave flumes," Journal of Fluids and Structures, vol. 7, no. 3, pp. 253-268, 1993.

[28] W. H. Press, B. P. Flannery, S. A. Teukolsky, and W. T. Vetterling, Numerical Recipes, Cambridge University Press, Cambridge, UK, 1986.

[29] W. Sulisz and M. Paprota, "Generation and propagation of transient nonlinear waves in a wave flume," Coastal Engineering, vol. 55, no. 4, pp. 277-287, 2008. 


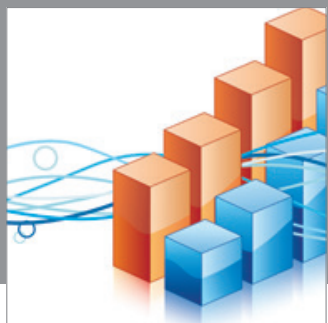

Advances in

Operations Research

mansans

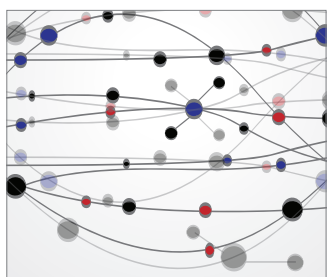

The Scientific World Journal
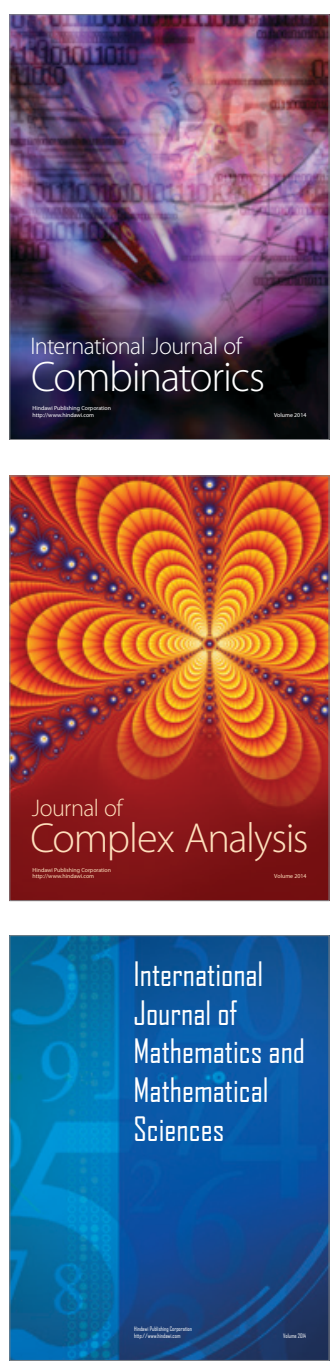
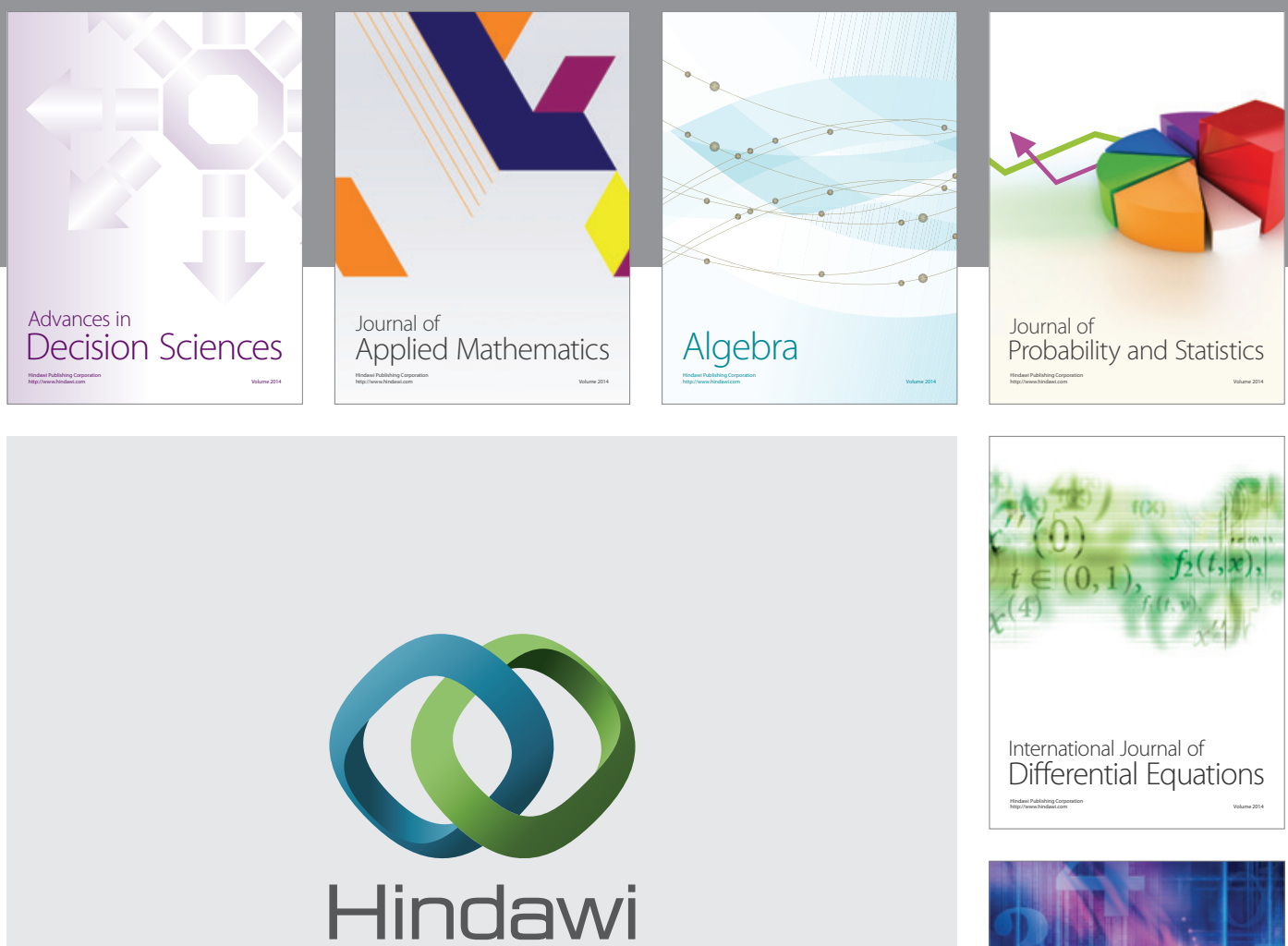

Submit your manuscripts at http://www.hindawi.com
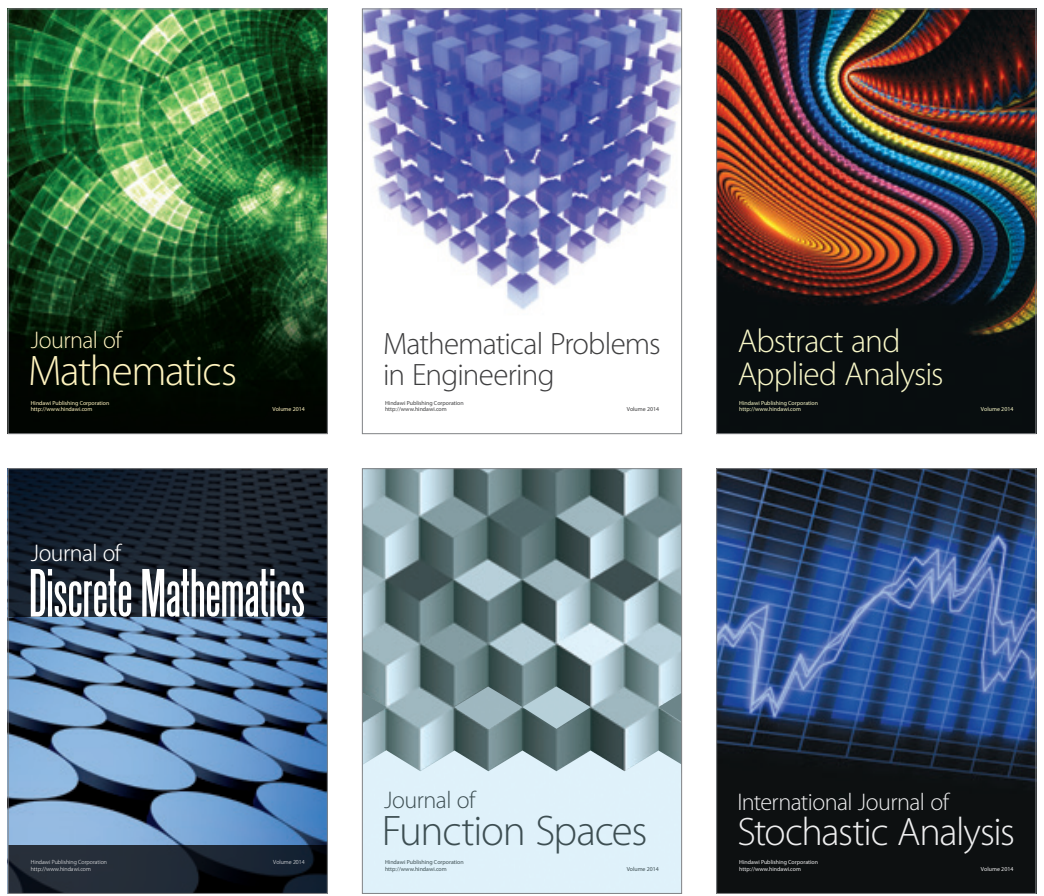

Journal of

Function Spaces

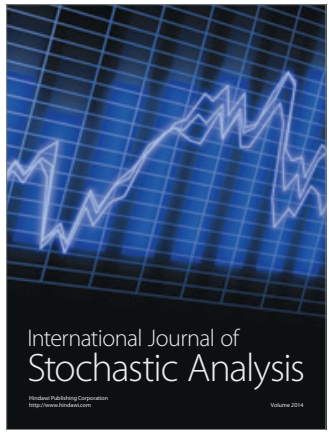

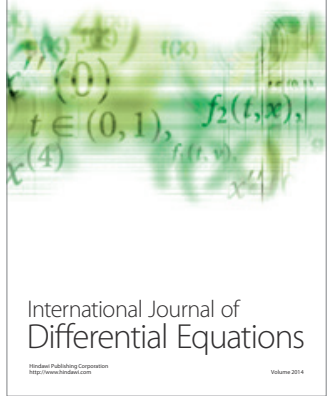
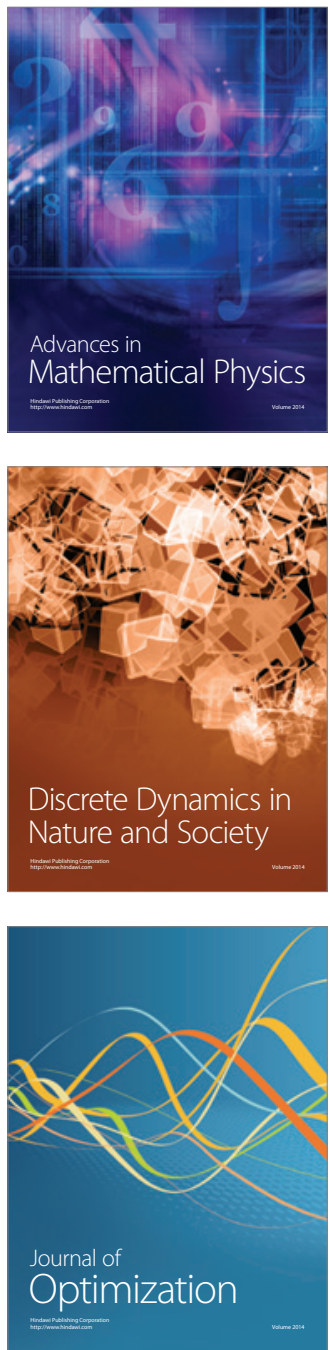